\title{
Vector spaces and the Petersen graph*
}

\author{
Marcelo H. de Carvalho ${ }^{\dagger}$ \\ UFMs, Campo Grande, Brasil \\ C. H. C. Little \\ Massey University, Palmerston North, New Zealand \\ Submitted: Aug 21, 2006; Accepted: Dec 17, 2007; Published: Jan 1, 2008 \\ Mathematics Subject Classification: 05C70
}

\begin{abstract}
It is shown that a matching covered graph has an ear decomposition with no more than one double ear if and only if there is no set $S$ of edges such that $|S \cap A|$ is even for every alternating circuit $A$ but $|S \cap C|$ is odd for some even circuit $C$. Two proofs are presented. The first uses vector spaces and the second is constructive. Some applications are also given.
\end{abstract}

\section{Introduction}

Look at the Petersen graph drawn below. The set $S=\left\{e_{2}, e_{4}, e_{15}\right\}$ of edges has the following property: $|S \cap A|$ is even for each alternating circuit $A$ but there is an even circuit $C$ (such as $\left\{e_{2}, e_{3}, e_{4}, e_{10}, e_{15}, e_{7}\right\}$ ) for which $|S \cap C|$ is odd. Bricks for which the underlying simple graph is the Petersen graph are the only bricks that have such a set $S$. This paper reveals why. We present two arguments. The first uses vector spaces, and is based on Lovász's formula for the dimension of the linear space over $G F(2)$ generated by the 1-factors of a matching covered graph. The second argument constructs $S$ in those matching covered graphs in which it exists.

We now present the relevant definitions, notation and background. The vertex and edge sets of a graph $G$ are denoted by $V G$ and $E G$ respectively. A 1-factor of $G$ is the edge set of a regular spanning subgraph of degree 1 . A connected graph is matching covered if every edge has a 1-factor containing it. Any such graph with more than two vertices is necessarily 2-connected. A graph $G$ is said to be bicritical if $G-\{u, v\}$ has a 1-factor for

\footnotetext{
*Supported by ProNEx - FAPESP (proc. 2003/09925-5), CNPq (proc. 478470/2006-1, 62.0123/04-4, 620171/2006-5 and 471745/2007-3) and FUNDECT-MS.

${ }^{\dagger}$ Supported by CNPq, Brasil.

${ }^{\ddagger}$ Work done during this author’s visit to UFMS, Brasil, in 2006 with support from CNPq.
} 


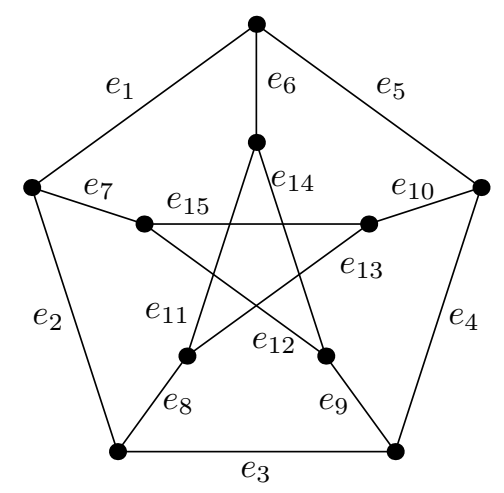

Figure 1: The Petersen graph

any two vertices $u$ and $v$. Such a graph must be matching covered and, moreover, cannot be bipartite if it has more than two vertices. A 3-connected bicritical graph is a brick. Any graph with the Petersen graph as its underlying simple graph furnishes an example of a brick. Now let $G$ be a matching covered bipartite graph with bipartition $\{X, Y\}$. We say that $G$ is a brace if $G-\{w, x, y, z\}$ has a 1-factor whenever $w$ and $x$ are distinct vertices of $X$ and $y$ and $z$ are distinct vertices of $Y$.

Any matching covered graph may be subjected to a decomposition procedure, due to Lovász [6]. It involves the concept of a tight cut. If $X$ is a set of vertices, then we write $\bar{X}=V G-X$. We let $\nabla X$ be the set of edges joining a vertex of $X$ to a vertex of $\bar{X}$. Thus $\nabla X=\nabla \bar{X}$. The set $\nabla X$ is called a cut of $G$. Its shores are $G[X]$ and $G[\bar{X}]$. We sometimes write $\nabla X=[X, \bar{X}]$. A cut is trivial if either shore has only one vertex.

Since $G$ is matching covered, $|V G|$ is even. Thus if $|X|$ is odd, then so is $|\bar{X}|$. In this case each 1-factor must contain an odd number of edges of $\nabla X$. If $|\nabla X \cap F|=1$ for each 1-factor $F$, then the cut $\nabla X$ is said to be tight. It has been shown by Lovász [6] that bricks and braces are the only matching covered graphs in which each tight cut is trivial.

Given a cut $T=\nabla X$, we may form two new graphs by contracting the respective shores of $T$ to single vertices. We denote by $G\{X ; \bar{x}\}$ the graph obtained from $G$ by contracting $\bar{X}$ to a single vertex $\bar{x}$. Similarly $G\{\bar{X} ; x\}$ denotes the graph formed by contracting $X$ to a single vertex $x$. These two graphs are called the $T$-contractions of $G$. They may be written more briefly as $G\{X\}$ and $G\{\bar{X}\}$, respectively, if no ambiguity emerges.

Now suppose that $T$ is a non-trivial tight cut in a matching covered graph. Then both $T$-contractions are also matching covered. We may repeat this process of finding non-trivial tight cuts and taking their contractions inductively until we are left with a family of graphs that lack non-trivial tight cuts. These graphs are bricks or braces. The process just described is referred to as tight cut decomposition. Lovász [6] has shown that the list of bricks and braces emerging from this procedure is uniquely determined up to multiplicities of edges. At least one brick emerges from the decomposition if and only if the graph is not bipartite.

The number of bricks plays an important role in the theory, not least because it is 
relevant to the calculation of the dimension of the linear space of the graph. Each vector space considered in this paper is over the field $G F(2)$, has sets of edges as vectors and has symmetric difference as the addition operation. The linear space, $\mathcal{L}(G)$, of a graph $G$ is the space spanned by the 1-factors. Lovász's formula for the dimension of $\mathcal{L}(G)$ depends on the number of bricks resulting from the decomposition procedure, except that bricks whose underlying simple graph is isomorphic to the Petersen graph are to be counted twice. Accordingly we let $b(G)$ be the total number of bricks resulting from the decomposition procedure applied to a matching covered graph $G$ and we let $p(G)$ be the number of those bricks whose underlying simple graph is isomorphic to the Petersen graph. We are then interested in the number $b(G)+p(G)$. Its relevance to the computation of $\operatorname{dim} \mathcal{L}(G)$ is shown in the following theorem of Lovász [6].

Theorem 1.1 For a matching covered graph $G$,

$$
\operatorname{dim} \mathcal{L}(G)=|E G|-|V G|+2-b(G)-p(G) .
$$

This formula bears a striking resemblance to that for the dimension of the cycle space, $\mathcal{C}(G)$, of $G$, which is the space spanned by the circuits. (Throughout this paper, paths and circuits are taken to be sets of edges.) Indeed, since $G$ is connected we have

$$
\operatorname{dim} \mathcal{C}(G)=|E G|-|V G|+1
$$

These spaces therefore have equal dimension if $b(G)+p(G)=1$.

For our purposes, a subspace of the cycle space, called the even space, is of particular importance. The even space, $\mathcal{E}(G)$, of $G$ is the subspace of $\mathcal{C}(G)$ spanned by the even circuits (those of even length). For bipartite graphs these spaces are the same, but for graphs that are not bipartite we have the following theorem. (See [4], for example.)

Theorem 1.2 For a 2-connected non-bipartite graph $G$,

$$
\operatorname{dim} \mathcal{E}(G)=\operatorname{dim} \mathcal{C}(G)-1
$$

The even space, in turn, has an important subspace called the alternating space and denoted by $\mathcal{A}(G)$. This is the subspace spanned by the alternating circuits. (A circuit is alternating if it is a symmetric difference of two 1-factors. More specifically, it is said to be alternating with respect to either of those 1-factors.)

In the next section we derive the existence or otherwise of the set $S$ described earlier by relating the dimensions of the spaces $\mathcal{L}(G), \mathcal{C}(G), \mathcal{E}(G)$ and $\mathcal{A}(G)$.

\section{$2 \quad$ An argument using vector spaces}

Every generating set for a vector space over any field includes a basis. In particular, we have the following theorem. 
Theorem 2.1 Let $G$ be a graph with a 1-factor. Then $\mathcal{L}(G)$ has a basis consisting of 1 -factors.

We have already seen in Theorem 1.2 how the dimensions of the cycle and even spaces are related for a 2-connected non-bipartite graph. We now derive an analogous result for the linear and alternating spaces in a graph with a 1-factor.

Theorem 2.2 For a graph $G$ with a 1-factor,

$$
\operatorname{dim} \mathcal{A}(G)=\operatorname{dim} \mathcal{L}(G)-1 .
$$

Proof: Let $\left(F_{0}, F_{1}, \ldots, F_{n}\right)$ be a basis $\mathcal{B}$ for $\mathcal{L}(G)$, where $F_{0}, F_{1}, \ldots, F_{n}$ are 1 -factors, and let

$$
\mathcal{A}=\left(F_{0}+F_{1}, F_{0}+F_{2}, \ldots, F_{0}+F_{n}\right) .
$$

We shall show that $\mathcal{A}$ is a basis for $\mathcal{A}(G)$.

First we consider a non-empty set

$$
\left\{F_{0}+F_{i_{1}}, F_{0}+F_{i_{2}}, \ldots, F_{0}+F_{i_{m}}\right\}
$$

of components of $\mathcal{A}$. Observe that either

$$
\sum_{j=1}^{m}\left(F_{0}+F_{i_{j}}\right)=\sum_{j=1}^{m} F_{i_{j}}
$$

or

$$
\sum_{j=1}^{m}\left(F_{0}+F_{i_{j}}\right)=F_{0}+\sum_{j=1}^{m} F_{i_{j}},
$$

depending on the parity of $m$. In either case the linear independence of $\mathcal{B}$ is contradicted if we assume that

$$
\sum_{j=1}^{m}\left(F_{0}+F_{i_{j}}\right)=\emptyset .
$$

We infer that $\mathcal{A}$ is linearly independent.

Next, let $C$ be an alternating circuit. Then $C=X+Y$ for some 1-factors $X$ and $Y$. Both $X$ and $Y$ are sums of components of $\mathcal{B}$. Since every 1-factor contains an odd number of edges incident on any vertex, each of these sums must have an odd number of summands. Therefore $C$ is a sum of an even number of components of $\mathcal{B}$. Let these components be $F_{l_{1}}, F_{l_{2}}, \ldots, F_{l_{2 k}}$. Then

$$
C=\sum_{j=1}^{2 k} F_{l_{j}}=\sum_{j=1}^{2 k}\left(F_{0}+F_{l_{j}}\right) .
$$

As $F_{0}+F_{0}=\emptyset$, the components of $\mathcal{A}$ span $\mathcal{A}(G)$. The theorem follows.

Let $G$ be a matching covered graph. If $b(G)+p(G)=0$, then $G$ is bipartite and $\mathcal{A}(G)=\mathcal{E}(G)$, by Theorem 1.1 and Theorem 2.2. Consequently every even circuit is a sum of alternating circuits. 
If $b(G)+p(G)=1$ then by Theorem 1.1 we have

$$
\operatorname{dim} \mathcal{L}(G)=\operatorname{dim} \mathcal{C}(G)
$$

and so

$$
\operatorname{dim} \mathcal{A}(G)=\operatorname{dim} \mathcal{E}(G)
$$

by Theorem 1.2 and Theorem 2.2. Once again, in this case $\mathcal{A}(G)=\mathcal{E}(G)$ and every even circuit is a sum of alternating circuits.

On the other hand, if $b(G)+p(G)>1$ then by the same argument we have

$$
\operatorname{dim} \mathcal{A}(G)<\operatorname{dim} \mathcal{E}(G) .
$$

Thus $\mathcal{A}(G)$ is a proper subspace of $\mathcal{E}(G)$ and there must be an even circuit that is not a sum of alternating circuits.

In summary, we have the following theorem.

Theorem 2.3 Let $G$ be a matching covered graph. Then $b(G)+p(G)>1$ if and only if there is an even circuit that is not a sum of alternating circuits.

Let $A_{1}, A_{2}, \ldots, A_{m}$ be the alternating circuits in a matching covered graph $G$. If $C$ is a circuit that is a sum of alternating circuits, then there exist constants $x_{1}, x_{2}, \ldots, x_{m}$ such that $x_{i} \in\{0,1\}$ for all $i$ and

$$
C=\sum_{i=1}^{m} x_{i} A_{i} .
$$

Now let $E G=\left\{e_{1}, e_{2}, \ldots, e_{n}\right\}$, and for each $j$ let $c_{j}=1$ if $e_{j} \in C$ and let $c_{j}=0$ otherwise. Similarly for each relevant $i$ and $j$ let $a_{i j}=1$ if $e_{j} \in A_{i}$ and let $a_{i j}=0$ otherwise. From (1) we have the equation

$$
c_{j}=\sum_{i=1}^{m} a_{i j} x_{i}
$$

for each $j$. In fact, $C$ is a sum of alternating circuits if and only if these $n$ equations have a solution for $x_{1}, x_{2}, \ldots, x_{m}$ over $G F(2)$. By applying the necessary and sufficient condition for the existence of a solution for a system of linear equations, we obtain the following corollaries.

Corollary 2.4 Let $G$ be a matching covered graph. Then $b(G)+p(G)>1$ if and only if there exists a set $S$ of edges such that $|S \cap A|$ is even for each alternating circuit $A$ but $|S \cap C|$ is odd for some even circuit $C$.

For brevity we shall refer to a set $S$ of edges in a matching covered graph as complicating if $|S \cap A|$ is even for each alternating circuit $A$ but $|S \cap C|$ is odd for some even circuit $C$. A matching covered graph is said to be a near brick if $b(G)=1$. In particular, any brick is a near brick.

Corollary 2.5 Let $G$ be a near brick. Then the unique brick in the decomposition of $G$ into bricks and braces has the Petersen graph as its underlying simple subgraph if and only if there exists a complicating set of edges. 


\section{A constructive argument}

We now consider how to obtain the results in the previous section constructively. The key tool here is another decomposition of a matching covered graph, called the ear decomposition. An ear in a graph $G$ is a maximal path whose internal vertices are of degree 2 in $G$. If $P$ is an ear in $G$ and $H=G[E G-P]$, then we say that $G$ is obtained from $H$ by a single ear adjunction. Likewise, let $P$ and $Q$ be vertex-disjoint ears of $G$. If $H=G[E G-(P \cup Q)]$, then $G$ is obtained from $H$ by a double ear adjunction. An ear decomposition of a matching covered graph $G$ is a sequence $G_{0}, G_{1}, \ldots, G_{n}$ of matching covered subgraphs such that $G_{0}=K_{2}, G_{n}=G$ and, for all $i>0, G_{i}$ is obtained from $G_{i-1}$ by a single or double ear adjunction.

The following theorem is proved in [7]. (See also [5] and [8].)

Theorem 3.1 Every matching covered graph has an ear decomposition.

An ear decomposition of a matching covered graph is an invaluable tool, often facilitating the use of induction. It is especially helpful if the decomposition is chosen so that the number of steps requiring a double ear adjunction is minimised. Thus we seek an ear decomposition that minimises the number of double ear adjunctions, that is, the number of values of $i>0$ such that $G_{i}$ is obtained from $G_{i-1}$ by a double ear adjunction. Such an ear decomposition is said to be optimal. In particular, if $G_{i}$, for some $i>0$, is obtained from $G_{i-1}$ by the adjunction of ears $P$ and $Q$ in an optimal decomposition, then neither $G\left[E G_{i}-P\right]$ nor $G\left[E G_{i}-Q\right]$ is matching covered. In this case, any alternating circuit in $G_{i}$ that includes $P$ must also include $Q$.

The number of double ear adjunctions required in an optimal ear decomposition has been found by de Carvalho, Lucchesi and Murty in [3] to be $b(G)+p(G)$. By Theorem 1.1 and Theorem 2.2, this number is $\operatorname{dim} \mathcal{C}(G)-\operatorname{dim} \mathcal{A}(G)$. Thus we have the following theorem.

Theorem 3.2 The number of double ear adjunctions in an optimal ear decomposition of a matching covered graph $G$ is $\operatorname{dim} \mathcal{C}(G)-\operatorname{dim} \mathcal{A}(G)$.

Given an optimal ear decomposition $G_{0}, G_{1}, \ldots, G_{n}$ of a matching covered graph $G$, for each $i>0$ there is an alternating circuit $A_{i}$ in $G_{i}$ that includes the ears adjoined to $G_{i-1}$ in the construction of $G_{i}$. The alternating circuits $A_{1}, A_{2}, \ldots, A_{n}$ are described as basic. They are linearly independent, and it follows from Theorem 3.2 that there are just enough of them to span $\mathcal{A}(G)$. Accordingly we have the following corollary.

Corollary 3.3 Given an optimal ear decomposition of a matching covered graph $G$, any set of basic alternating circuits constitutes a basis for $\mathcal{A}(G)$.

Note that the basic alternating circuits alternate with respect to a fixed 1-factor $F$ such that $F \cap E G_{i}$ is a 1 -factor of $G_{i}$ for each $i$. Consequently each ear in the decomposition is of odd length. 
With these preliminaries out of the way, we are now ready for the construction of the complicating set $S$ of Corollary 2.4 or a direct proof that no such set exists. In the latter case, it suffices to show that the alternating and even spaces of the graph coincide. Throughout this discussion, we will be contemplating an optimal ear decomposition of a matching covered graph $G$, and we will denote by $H$ the penultimate graph of the decomposition.

We begin with the following theorem.

Theorem 3.4 If $H$ has no complicating set of edges and $G$ is obtained from $H$ by a single ear adjunction, then $G$ has no complicating set of edges.

Proof: Let $G$ be obtained from $H$ by the adjunction of an ear $P$. This ear is a path in some alternating circuit $A$ of $G$. Now consider an even circuit $C$ of $G$. If $C \subseteq E H$ then $C$ is a sum of alternating circuits in $H$ and these are also alternating in $G$. In the remaining case $P \subset C$. Thus $A+C$ is the union of disjoint even circuits in $H$. Hence there are alternating circuits $A_{1}, A_{2}, \ldots, A_{n}$ in $H$ (and therefore in $G$ ) such that

$$
A+C=\sum_{i=1}^{n} A_{i} .
$$

Consequently

$$
C=A+\sum_{i=1}^{n} A_{i}
$$

as required.

This theorem is applicable in the case where no double ear adjunctions occur in an ear decomposition of $G$. This case arises precisely when $G$ is bipartite. Thus we have the following corollary.

Corollary 3.5 Matching covered bipartite graphs have no complicating set of edges.

It follows immediately that if $S$ is a set of edges of a bipartite matching covered graph $G$ and $|S \cap A|$ is even for each alternating circuit $A$, then $|S \cap C|$ is even for each circuit $C$.

Next we deal with the case where the last ear adjunction is the only double one. This case arises precisely when $H$ is bipartite but $G$ is not.

Theorem 3.6 Let $G$ be a non-bipartite matching covered graph obtained from a bipartite matching covered subgraph $H$ by a (double) ear adjunction. Then $G$ has no complicating set of edges.

Proof: Let $G$ be obtained from $H$ by the adjunction of ears $P$ and $Q$. Thus every alternating circuit in $G$ including $P$ or $Q$ must include them both. In addition, $G[E G-P]$ and $G[E G-Q]$ are not bipartite, and so any even circuit including $P$ or $Q$ must also include them both. It therefore follows, as in the proof of Theorem 3.4, that every even 
circuit is a sum of alternating circuits, since each even circuit $C$ satisfies $C \subseteq E H$ or $P \cup Q \subset C$.

Suppose next that just one double ear adjunction occurs during an optimal ear decomposition of $G$ but the last ear adjunction is single. It follows from Theorem 3.6, by applying Theorem 3.4 inductively, that every even circuit of $G$ is a sum of alternating circuits.

Next we consider the case where $H$ is not bipartite and $G$ is obtained from $H$ by a double ear adjunction. This double ear adjunction is at least the second of the decomposition.

Theorem 3.7 If $G$ is obtained from a non-bipartite graph $H$ by a double ear adjunction, then $G$ has a complicating set of edges.

Proof: Let $G$ be obtained from $H$ by the adjunction of ears $P$ and $Q$. Thus every alternating circuit of $G$ including $P$ or $Q$ also includes the other. On the other hand, the non-bipartite graph $H$ has an odd circuit $D$. Being matching covered, $H$ is 2-connected. Thus by Menger's theorem there are vertex-disjoint paths $X$ and $Y$ in $H$, of minimal length, joining the respective ends of $P$ to distinct vertices $x$ and $y$ of $D$. Let $R=$ $X \cup P \cup Y$. The odd circuit $D$ includes a path $Z$, joining $x$ and $y$, of the same parity as $|R|$. By the minimality of $X$ and $Y$, we find that $R \cup Z$ is a circuit $C$, and the choice of $Z$ ensures that $C$ is even. Now let $S=P \cup Q$. Then $|S \cap A|$ is even for each alternating circuit $A$ but $|S \cap C|$ is odd. Hence $S$ is the required complicating set.

Finally we deal with the case where at least two double ear adjunctions occur in the decomposition of $H$ but $G$ is obtained from $H$ by a single ear adjunction. Here $H$ has a complicating set of edges. In the proof of the following theorem we make essential use of the fact that the ear decomposition is optimal.

Theorem 3.8 Suppose that $H$ has a complicating set of edges and that $G$ is obtained from $H$ by a single ear adjunction. Then $G$ has a complicating set of edges.

Proof: Let $S$ be a complicating set of edges in $H$. Note that $|S \cap C|$ is odd for some even circuit $C$ in $H$. Let $G$ be constructed from $H$ by the adjunction of a single ear $P$. Then $P \subset A$ for some alternating circuit $A$ of $G$. Since the ear decomposition is optimal, by Corollary 3.3 there is a basis $\mathcal{B}$ for $\mathcal{A}(H)$ such that $\mathcal{B}^{\prime}=\mathcal{B} \cup\{A\}$ is a basis for $\mathcal{A}(G)$.

Suppose that $|A \cap S|$ is even. By assumption this property holds also for each alternating circuit in $H$ and hence for every member of $\mathcal{B}^{\prime}$. Therefore it holds for each member of $\mathcal{A}(G)$ and in particular for each alternating circuit that includes $P$. Thus if one alternating circuit of $G$ that includes $P$ has an intersection with $S$ of even cardinality, then all such alternating circuits do. It follows that if one such alternating circuit has an intersection with $S$ of odd cardinality, then all of them do.

We conclude that the parity of $|A \cap S|$ is well defined. Let $S^{\prime}=S$ if this parity is even; otherwise let $S^{\prime}=S \cup P$. Since $|P|$ is odd, $\left|A \cap S^{\prime}\right|$ is even. This property therefore 
holds for every member of $\mathcal{B}^{\prime}$ and consequently for every alternating circuit in $G$, since $S^{\prime} \cap E H=S$. For the same reason, $\left|C \cap S^{\prime}\right|$ is odd for the even circuit $C$ of $G$.

As an example, we refer once again to Figure 1. There is an optimal ear decomposition defined by the following sets of ears, in order:

$$
\left(\left\{\left\{e_{8}\right\}\right\},\left\{\left\{e_{3}, e_{9}, e_{12}, e_{7}, e_{1}, e_{6}, e_{11}\right\}\right\},\left\{\left\{e_{5}, e_{10}, e_{13}\right\},\left\{e_{14}\right\}\right\},\left\{\left\{e_{4}\right\},\left\{e_{15}\right\}\right\},\left\{\left\{e_{2}\right\}\right\}\right) .
$$

The last double ear is $\left\{\left\{e_{4}\right\},\left\{e_{15}\right\}\right\}$, and it is followed only by the single ear $\left\{\left\{e_{2}\right\}\right\}$. Moreover $\left\{e_{2}, e_{8}, e_{13}, e_{10}, e_{4}, e_{9}, e_{12}, e_{7}\right\}$ is an alternating circuit that contains $e_{2}$ and just one of $e_{4}$ and $e_{15}$. According to our construction, we therefore obtain the complicating set $S=\left\{e_{2}, e_{4}, e_{15}\right\}$ mentioned before. Note, however, that this is not the only possible choice for $S$. Any pentagon supplies another example.

\section{Applications}

We conclude this paper with some examples of theorems in whose proofs the concept of a complicating set is useful. For brevity, we shall refer to a graph as ordinary if it has no complicating set of edges. As we have already seen, this is the case if and only if every even circuit is a sum of alternating circuits (with respect to some fixed 1-factor $F$ ).

Theorem 4.1 Let $T=[X, \bar{X}]$ be a tight cut in a matching covered graph $G$. Let $G\{X ; \bar{x}\}$ and $G\{\bar{X} ; x\}$ be the two T-contractions. If $G$ is ordinary then so are $G\{X\}$ and $G\{\bar{X}\}$.

Proof: Let $C$ be an even circuit in $G\{X ; \bar{x}\}$. Two cases are possible, according to whether $C$ passes through $\bar{x}$.

Case I: Suppose first that $C$ does not pass through $\bar{x}$. Then $C$ is an even circuit in $G$. Accordingly $C$ is a sum of alternating circuits $A_{1}, A_{2}, \ldots, A_{n}$ in $G$ with respect to some 1-factor $F$.

Fix $i \in\{1,2, \ldots, n\}$. Suppose that $A_{i} \cap T=\emptyset$. Then either $A_{i} \subseteq E G\{X\}$ or $A_{i} \cap E G\{X\}=\emptyset$. In the former case $A_{i}$ is an alternating circuit in $G\{X\}$, since the tightness of $T$ implies that $F \cap E G\{X\}$ is a 1-factor of $G\{X\}$. If $A_{i} \cap T \neq \emptyset$, then $\left|A_{i} \cap T\right|=2$ and $\left|A_{i} \cap T \cap F\right|=1$. It follows again that $A_{i} \cap E G\{X\}$ is an alternating circuit in $G\{X\}$ with respect to $F$.

Thus in all cases we find that $A_{i} \cap E G\{X\}$, if non-empty, is an alternating circuit in $G\{X\}$. The sum of these alternating circuits is $C$.

Case II: Suppose $C$ passes through $\bar{x}$. Let $e$ and $f$ be the edges of $C$ incident on $\bar{x}$. Let $u$ and $v$, respectively, be the ends of these edges in $\bar{X}$. Let $M_{e}$ and $M_{f}$ be perfect matchings of $G$ containing $e$ and $f$, respectively.

Let $D$ be the circuit of $M_{e}+M_{f}$ containing $e$. Since $T$ is tight, $M_{e} \cap T=\{e\}$ and $M_{f} \cap T=\{f\}$, and it follows that $f \in D$. Now, the segment of $D-\{e, f\}$ contained in $G[\bar{X}]$ is an even path $P$ joining $u$ and $v$. Thus $C \cup P$ is a circuit of even length in $G$. 
We infer that $C \cup P$ is a sum of alternating circuits $A_{1}, A_{2}, \ldots, A_{n}$ in $G$ with respect to $F$. As in Case I, their non-empty intersections with $E G\{X\}$ are alternating circuits in $G\{X\}$ with $\operatorname{sum} C$.

We have now shown that $G\{X\}$ is ordinary. The proof that $G\{\bar{X}\}$ is ordinary is similar.

Theorem 4.2 Let $T=[X, \bar{X}]$ be a tight cut in a matching covered graph $G$. Suppose there exists a circuit $C$ in $G$ such that $|C \cap E G[X]|$ and $|C \cap E G[\bar{X}]|$ are both odd. Then $G$ is not ordinary.

Proof: Were $G$ ordinary, the even circuit $C$ would be a sum of alternating circuits. But consider an alternating circuit $A$ with respect to a fixed 1-factor $F$. If $A \cap T=\emptyset$ then $A \subseteq E G[X]$ or $A \subseteq E G[\bar{X}]$. The remaining possibility is that $|A \cap T|=2$ and $|A \cap F \cap T|=1$. In every case it follows that $|A \cap E G[X]|$ is even. However $|C \cap E G[X]|$ is odd. Hence $C$ cannot be a sum of alternating circuits and $G$ is not ordinary.

Theorem 4.3 If neither $G[X]$ nor $G[\bar{X}]$ is bipartite, then $G$ is not ordinary.

Proof: By hypothesis, $b(G)+p(G) \geq 2$, and so the result follows from Theorem 2.3.

The proof of the next theorem is facilitated by the following lemma, which concerns binary sequences. Let $n$ be a positive integer. We define a binary sequence of length $n$ to be an ordered $n$-tuple of zeros and ones. The binary sequence $\left(a_{1}, a_{2}, \ldots, a_{n}\right)$ is usually written more compactly as $a_{1} a_{2} \ldots a_{n}$. Now let $B$ be a binary sequence of even length, $2 k$. We may write this binary sequence as $b_{1} c_{1} b_{2} c_{2} \ldots b_{k} c_{k}$. For each $j \in\{1,2, \ldots, k\}$, define

$$
m_{j}(B)= \begin{cases}1 & \text { if } b_{j}=c_{j} \\ 0 & \text { otherwise }\end{cases}
$$

and let

$$
m(B)=\sum_{j=1}^{k} m_{j}(B)
$$

Lemma 4.4 Let $B$ be a binary sequence such that the number of ones is congruent modulo 4 to the number of zeros. Then $m(B)$ is even.

Proof: We begin with the case where $B$ is of the form $00 \ldots 011 \ldots 1$. We may write the number of zeros as $2 q+r$ for some non-negative integers $q$ and $r$, where $r \in\{0,1\}$. Then the number of ones is $2 q+r+4 s$ for some integer $s$. In this case it follows that

$$
m(B)=q+(q+2 s)=2 q+2 s,
$$

which is even.

Any other binary sequence $B^{\prime}$ with the same number of zeros as $B$ and the same number of ones as $B$ is obtained by a permutation of $B$. It therefore suffices to show 
that $m\left(B^{\prime}\right)$ has the parity of $m(B)$ whenever $B^{\prime}$ is obtained by a transposition of two consecutive components of $B$. Let

$$
B=b_{1} c_{1} b_{2} c_{2} \ldots b_{k} c_{k}
$$

There is no loss of generality in considering just the cases where

$$
B^{\prime}=c_{1} b_{1} b_{2} c_{2} b_{3} c_{3} \ldots b_{k} c_{k}
$$

or

$$
B^{\prime}=b_{1} b_{2} c_{1} c_{2} b_{3} c_{3} b_{4} c_{4} \ldots b_{k} c_{k} .
$$

In the former case $m\left(B^{\prime}\right)=m(B)$. In the latter case, the same equation holds if $c_{1}=b_{2}$. If $c_{1} \neq b_{2}$, however, then we have $m_{1}\left(B^{\prime}\right)=1-m_{1}(B), m_{2}\left(B^{\prime}\right)=1-m_{2}(B)$ and $m_{j}\left(B^{\prime}\right)=m_{j}(B)$ for all $j>2$. Hence

$$
m\left(B^{\prime}\right)=2-m_{1}(B)-m_{2}(B)+\sum_{j=3}^{k} m_{j}(B) \equiv m(B) \quad(\bmod 2) .
$$

In every case we have

$$
m\left(B^{\prime}\right) \equiv m(B) \quad(\bmod 2),
$$

and the result follows immediately.

Theorem 4.5 Let $\nabla X$ be a bond in a near brick $G$, and suppose that its shores $G[X]$ and $G[\bar{X}]$ are 2-connected and non-bipartite. Suppose that for any two 1-factors $F_{1}$ and $F_{2}$ of $G$ we have

$$
\left|F_{1} \cap \nabla X\right| \equiv\left|F_{2} \cap \nabla X\right| \quad(\bmod 4) .
$$

Then the unique brick in the decomposition of $G$ into bricks and braces has the Petersen graph as its underlying simple graph.

Proof: Fix a 1-factor $F$ of $G$ and let $A$ be an alternating circuit of the form $F+F^{\prime}$ for some 1-factor $F^{\prime}$ of $G$. If $A \cap \nabla X=\emptyset$, then $A \cap E G[X] \in\{\emptyset, A\}$. Hence $|A \cap G[X]|$ is even in this case. We shall therefore assume that $A \cap \nabla X \neq \emptyset$ and show that $|A \cap G[X]|$ is even under this hypothesis as well.

From $A$ we construct a binary sequence in the following manner: traverse $A$ in either sense, starting at any initial vertex, and record a 1 each time an edge of $F \cap \nabla X$ is encountered and a 0 each time an edge of $F^{\prime} \cap \nabla X$ is met. Let $r$ and $s$ denote the numbers of ones and zeros, respectively. Thus $r+s$ is even, so that $r \equiv s(\bmod 2)$.

Suppose that

$$
r \equiv s+2 \quad(\bmod 4)
$$

Then

$$
\left|F^{\prime} \cap \nabla X\right|=|F \cap \nabla X|-r+s \equiv|F \cap \nabla X|+2 \quad(\bmod 4),
$$

contrary to hypothesis. We infer that $r \equiv s(\bmod 4)$, and consequently that Lemma 4.4 is applicable. 
Now consider any maximal path $R$ included in $A \cap E G[X]$. There are just two edges in $A \cap \nabla X$ having an end in common with $R$; call them $x$ and $y$ respectively. Then $|R|$ is odd if and only if $x$ and $y$ are both in $F$ or both in $F^{\prime}$. By Lemma 4.4 we find that there are an even number of maximal paths $R$ included in $A \cap E G[X]$ that satisfy this condition. Hence $|A \cap E G[X]|$ is even. Since alternating circuits that alternate with respect to $F$ span $\mathcal{A}(G)$, we conclude that every alternating circuit has an intersection with $E G[X]$ of even cardinality.

Finally, choose independent edges $e$ and $f$ in $\nabla X$. Since $G[X]$ is 2-connected and not bipartite, there is a path $P$ in $G[X]$, of odd length, joining the ends of $e$ and $f$ in $X$. Similarly there is a corresponding odd path $Q$ in $G[\bar{X}]$ joining the ends of $e$ and $f$ in $\bar{X}$. Thus $P \cup Q \cup\{e, f\}$ is a circuit $C$, of even length, such that $|C \cap E G[X]|$ is odd.

The theorem now follows immediately from Corollary 2.5.

A graph $G$ is critical if $G-\{v\}$ has a 1 -factor for each vertex $v$.

Corollary 4.6 Let $G$ be a near brick with an odd cut $\nabla X=[X, \bar{X}]$. Suppose that $G[X]$ and $G[\bar{X}]$ are critical, 2-connected and non-bipartite, and that

$$
|F \cap E G[X]| \equiv \frac{|X|-1}{2}(\bmod 2)
$$

for all 1-factors $F$. Then the unique brick resulting from the decomposition of $G$ into bricks and braces has the Petersen graph as its underlying simple graph.

Proof: Let $e$ be an edge of $\nabla X$ joining vertices $u \in X$ and $v \in \bar{X}$. As $G[X]$ is critical, $G[X]-\{u\}$ has a 1-factor $F_{1}$. Similarly $G[\bar{X}]-\{v\}$ has a 1-factor $F_{2}$. Let $F=F_{1} \cup F_{2} \cup\{e\}$. Then $F$ is a 1-factor of $G$ such that $|F \cap \nabla X|=1$.

Now let $F^{\prime}$ be another 1-factor of $G$. By hypothesis,

$$
\left|F^{\prime} \cap E G[X]\right| \equiv|F \cap E G[X]| \quad(\bmod 2) .
$$

Therefore

$$
\left|F^{\prime} \cap \nabla X\right| \equiv|F \cap \nabla X| \quad(\bmod 4) .
$$

The result now follows from the theorem.

\section{Acknowledgments}

We thank C.L. Lucchesi and O. Lee for useful discussions. The second author also thanks the Federal University of Mato Grosso do Sul for its hospitality while the research was undertaken.

\section{References}

[1] M.H. de Carvalho, C.L. Lucchesi, U.S.R. Murty, On a Conjecture of Lovász Concerning Bricks. I. The Characteristic of a Matching Covered Graph, J. Combinatorial Theory B85 (2002), 94-136. 
[2] M.H. de Carvalho, C.L. Lucchesi, U.S.R. Murty, On a Conjecture of Lovász Concerning Bricks. II. Bricks of Finite Characteristic, J. Combinatorial Theory B85 (2002), $137-180$.

[3] M.H. de Carvalho, C.L. Lucchesi, U.S.R. Murty, Optimal ear decompositions of matching covered graphs and bases for the matching lattice, J. Combinatorial Theory B85 (2002), 59-93.

[4] M.A. Henning, C.H.C. Little, On the principal edge bipartition of a graph, Australasian J. Combinatorics 20 (1999), 91-96.

[5] C.H.C. Little, F. Rendl, Operations preserving the Pfaffian property of a graph, J. Austral. Math. Soc. A50 (1991), 248-257.

[6] L. Lovász, Matching structure and the matching lattice, J. Combinatorial Theory B43 (1987), 187-222.

[7] L. Lovász, M.D. Plummer, "Matching Theory", North-Holland, Amsterdam, 1986.

[8] Z. Szigeti, The two ear theorem on matching-covered graphs, J. Combinatorial Theory B74 (1998), 104-109. 